tissue counterpart of GCT of bone, a more aggressive neoplasm with risks of local recurrence and distant metastasis estimated to be $25 \%$ and $2 \%$, respectively. 3,4 According to the World Health Organization classification of soft tissue tumors, ${ }^{5}$ it is included in the group of so-called fibrohistiocytic lesions of indeterminate malignancy. ${ }^{1,2}$ Giant-cell tumor of skin or soft tissue must be distinguished from a direct extension or cutaneous metastasis of a conventional GCT of bone., ${ }^{1,2}$ The differential diagnosis also encompasses other skin lesions with multinucleated giant cells, including GCT of tendon sheath, cellular and/or ossifying dermatofibroma with osteoclastlike giant cells, plexiform fibrohistiocytic tumor, reparative giant-cell granuloma, nodular fasciitis, and brown tumor of hyperparathyroidism extending to soft tissues. It also encompasses a number of malignant tumors that may contain osteoclastlike giant cells, including carcinoma, melanoma, malignant fibrous histiocytoma, atypical fibroxanthoma, leiomyosarcoma, epithelioid sarcoma, and extraskeletal osteosarcoma. ${ }^{1,2,6}$ In general, GCT of skin or soft tissue demonstrates a favorable outcome, but it may show clinical persistence and/or recurrence if not completely excised. ${ }^{1,2}$ Metastasis to the lungs and lymph nodes is rarely reported. ${ }^{2}$

Telomerase is a ribonucleoprotein reverse transcriptase that adds hexameric telomere sequences (TTAGGG) to the ends of chromosomes. ${ }^{3,4,7}$ Its expression is repressed in most human somatic cells, leading to progressive loss of telomeres and chromosome shortening with successive cell divisions, so that cell division eventually ceases; senescence begins; and the cells ultimately undergo apoptosis or cell death. ${ }^{3,4,7}$ Telomerase activity has been described in up to $90 \%$ of human malignant neoplasms, including a number of cutaneous neoplasms. ${ }^{3,4,7}$ In this setting, high levels of telomerase may help to maintain stable telomere lengths and support the unregulated proliferation and immortality of tumor cells.

A number of studies have reported the nuclear expression of hTERT, which encodes the catalytic subunit of telomerase, in 35\% to $100 \%$ of GCT of bone; albeit at low and highly variable levels. ${ }^{3,4}$ In addition, positive immunohistochemical staining for hTERT correlates inversely with recurrence-free survival in GCT of bone and may be a useful prognostic marker in patients with this tumor. ${ }^{3}$ Granular cytoplasmic staining for hTERT can occasionally be found in human tumors. ${ }^{7,8}$ This finding may result from either alternative splicing of hTERT or a lack of posttranslational changes, with failure of a variant or unphosphorylated protein to translocate from the cytoplasm to the nucleus. ${ }^{8,9}$ Cytoplasmic hTERT could have extratelomeric roles, including enhancement of cellular

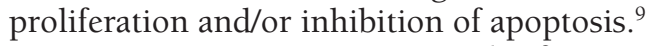

Herein, we report a rare example of GCT of skin with cytoplasmic staining for hTERT by immunohistochemical analysis. Lack of nuclear hTERT expression could explain the indolent clinical course associated with GCT of skin or soft tissues. The cytoplasmic role of hTERT and a possible extratelomeric effect remain to be investigated.
Author Affiliations: Department of Dermatology, University of Connecticut, Farmington.

Correspondence: Dr Murphy, Department of Dermatology, University of Connecticut Health Center, 21 South Rd, Farmington, CT 06030 (drmichaelmurphy@netscape .net).

Financial Disclosure: None reported.

1. Folpe AL, Morris RJ, Weiss SW. Soft tissue giant cell tumor of low malignant potential: a proposal for the reclassification of malignant giant cell tumor of soft parts. Mod Pathol. 1999;12(9):894-902.

2. Lentini M, Zuccalà V, Fazzari C. Polypoid giant cell tumor of the skin. Am J Dermatopathol. 2010;32(1):95-98.

3. Horvai AE, Kramer MJ, Garcia JJ, O'Donnell RJ. Distribution and prognostic significance of human telomerase reverse transcriptase (hTERT) expression in giant-cell tumor of bone. Mod Pathol. 2008;21(4):423-430.

4. Gebre-Medhin S, Broberg K, Jonson T, et al. Telomeric associations correlate with telomere length reduction and clonal chromosome aberrations in giant cell tumor of bone. Cytogenet Genome Res. 2009;124(2):121-127.

5. Fletcher CDM, Krishnan Unni K, Mertens F; The International Agency for Research on Cancer. Pathology and Genetics of Tumours of Soft Tissue and Bone: WHO Classification of Tumours. Geneva, Switzerland: World Health Organization; 2003

6. Wooff J, Werner D, Murphy J, Walsh N. Osteoclast-like giant cell reaction associated with cutaneous squamous cell carcinoma: a report of 2 cases and review of the literature. Am J Dermatopathol. 2009;31(3):282-287.

7. Olsen SH, Su LD, Thomas D, Fullen DR. Telomerase expression in sebaceous lesions of the skin. J Cutan Pathol. 2007:34(5):386-391.

8. Yan P, Benhattar J, Seelentag W, Stehle JC, Bosman FT. Immunohistochemical localization of hTERT protein in human tissues. Histochem Cell Biol. 2004; 121(5):391-397.

9. Lepreux S, Doudnikoff E, Aubert I, Bioulac-Sage P, Bloch B, Martin-Negrier ML. Cytoplasmic expression of human telomerase catalytic protein (hTERT) in neutrophils: an immunoelectron microscopy study. Ultrastruct Pathol. 2008; 32(5):178-183.

\section{Eruptive Lichen Planus Triggered by Acupuncture}

Report of a Case. We report the case of a 41-year-old, previously fit and healthy woman who developed a widespread eruption following acupuncture treatment for musculoskeletal back pain. Four days after the treatment, she noticed a pruritic papular eruption on her lower back, where the acupuncture needles had punctured her skin. The eruption then spread to her feet, wrists, and chest. She took no regular medications apart from levothyroxine for hypothyroidism and was penicillin allergic.

On examination, we found that she had a florid, widespread eruption on her trunk and limbs, coalescing at skin fold sites on her back. On closer inspection, we noted dense crops of flat-topped, polygonal, red-brown and violaceous papules and plaques at sites of acupuncture needle placement on her lower back (Figure 1). The eruption also involved her trunk, back, and limbs, with dense foci on her wrists and ankles (Figure 2). Her face, palms, and soles were spared. She had a florid white lacy pattern consistent with Wickham striae on her buccal mucosa, but her genital mucosa was spared.

The results of blood tests, including hepatitis $\mathrm{B}$ and $C$ serologic assays, were unremarkable. A 4-mm punch biopsy specimen showed a dense bandlike lymphocytic infiltrate in the upper dermis obscuring the dermoepidermal junction, with some scattered lymphocytes and occasional eosinophils extending to the epidermis. The histologic findings therefore confirmed the diagnosis of widespread eruptive lichen planus.
Michael Murphy, MD

Philip Kerr, MD 


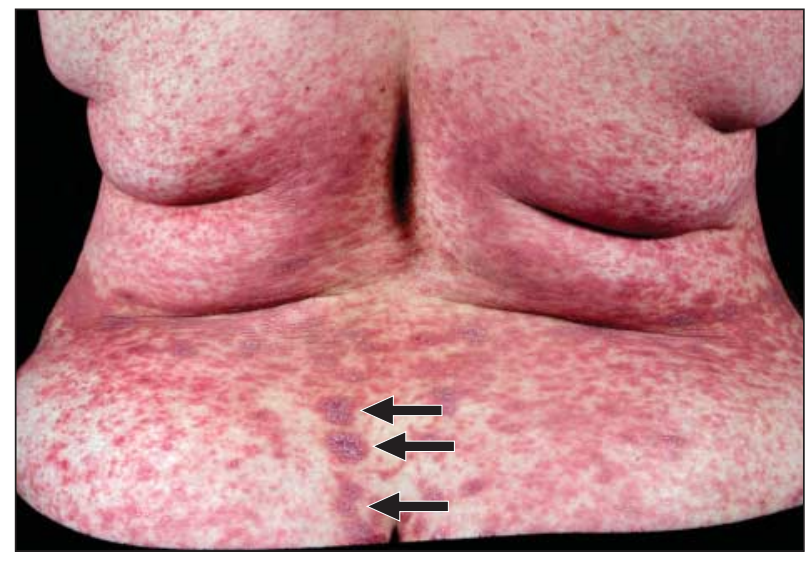

Figure 1. Crops of flat-topped, polygonal, red-brown and violaceous papules and plaques were originally centered at sites of acupuncture needle placement on the lower back (arrows) and then became more generalized.
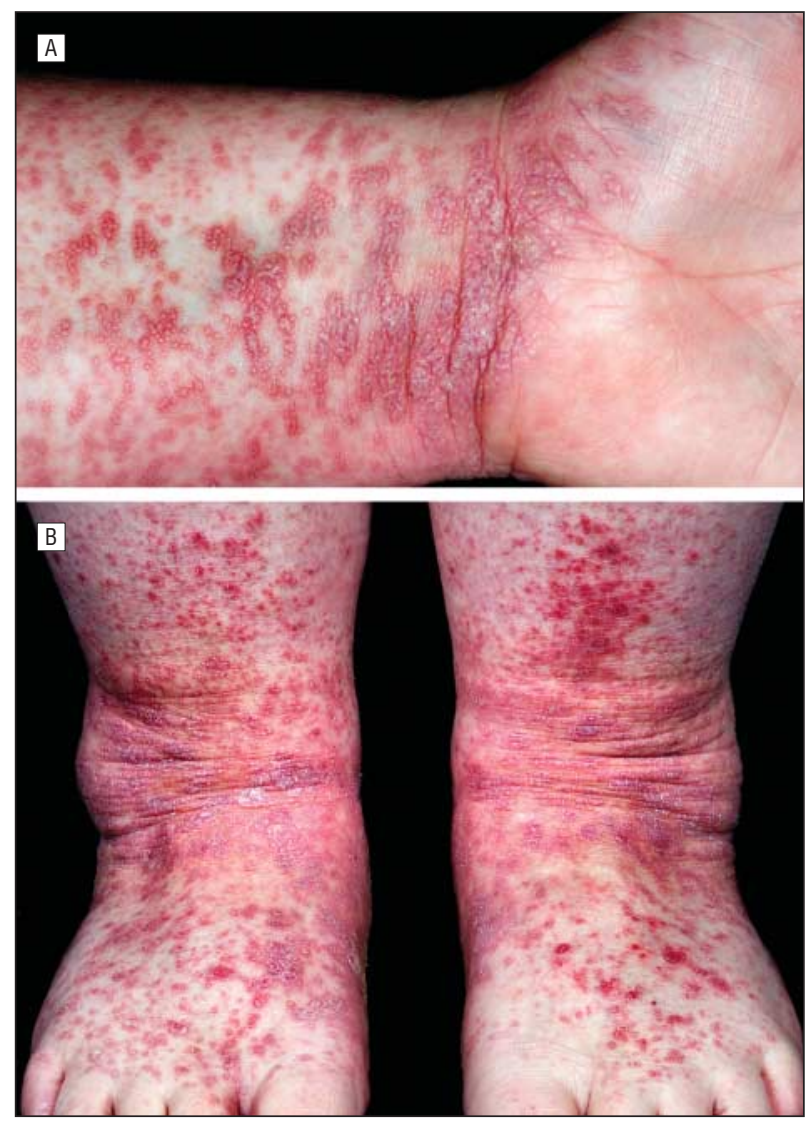

Figure 2. The intensely pruritic eruption was most dense at the wrists $(A)$ and ankles $(B)$.

Our patient was treated successfully with a 1-month course of tapering systemic corticosteroids and subsequently with UV-A phototherapy in combination with oral psoralen. Six months after presentation, her eruption had improved, leaving only postinflammatory hyperpigmentation in previously active sites, and no new lesions had developed.

Comment. Lichen planus is a moderately common skin disease, with a prevalence of around $0.5 \%$, occurring most commonly in middle-aged women. It is generally thought of as an autoimmune disorder, and there are associa- tions with HLA-A3, -A5, -B8, -Bw35, and -DR1. In some centers there has been a reported association with hepatitis B and C infection, although the strength of this link remains to be assessed.

The Koebner phenomenon has been described following acupuncture in a patient with existing psoriasis. ${ }^{1}$ In our case, however, the patient had no premorbid dermatosis and could not therefore koebnerize. We hypothesize that the acupuncture triggered the florid eruption either as an immune response to the skin trauma itself or potentially via a subclinical viral infection from inoculation. Lichen planus has been reported to occur following intramuscular injections ${ }^{2}$ and vaccination, ${ }^{3}$ especially hepatitis $\mathrm{B},{ }^{4}$ potentially secondary to the inactivated hepatitis virus itself, as opposed to koebnerization. To our knowledge, however, this is the first reported case of lichen planus being triggered by acupuncture.

The cause of lichen planus is unknown, and the eruptive and florid disease described here suggests that a cutaneous insult such as acupuncture can be enough to act as a trigger. We hypothesize that evoked koebnerization may produce a reactive immunologic cascade leading to widespread lichen planus.

Acupuncture is practiced widely for its analgesic properties and in a multitude of ailments such as depression, diabetes mellitus, arthritis, and irritable bowel syndrome. ${ }^{5}$ We suggest that those practicing or undergoing acupuncture be made aware of the potential of needle placement to evoke this immune-mediated dermatosis.

\section{John Fleming, BSc, MRCP \\ Salvador Diaz-Cano, MD, PhD, FRCPath Elisabeth Higgins, MA, FRCP}

Author Affiliations: Departments of Dermatology (Drs Fleming and Higgins) and Histopathology (Dr DiazCano), King's College Hospital, London, England.

Correspondence: Dr Fleming, Department of Dermatology, King's College Hospital London, London SE5 9RS, England (johnfleming@nhs.net).

Financial Disclosure: None reported.

1. Kirschbaum JO. Koebner phenomenon following acupuncture. Arch Dermatol. 1972;106(5):767.

2. Numata Y, Okuyama R, Tagami H, Aiba S. Linear lichen planus distributed in the lines of Blaschko developing during intramuscular triamcinolone acetonide therapy for alopecia areata multiplex. J Eur Acad Dermatol Venereol. 2008;22(11):1370-1372.

3. Akay BN, Arslan A, Cekirge S, Erkin G, Anadolu-Brasie R. The first reported case of lichen planus following inactivated influenza vaccination. J Drugs Dermatol. 2007;6(5):536-538.

4. Limas C, Limas CJ. Lichen planus in children: a possible complication of hepatitis B vaccines. Pediatr Dermatol. 2002;19(3):204-209.

5. The British Acupuncture Council. Research resources: fact sheets. http://www .acupuncture.org.uk/public-research.html. Accessed June 2010.

\section{Fixed Drug Eruption on the Penis Due to Oxcarbazepine}

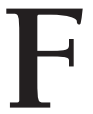

ixed drug eruption (FDE) is a common cutaneous drug adverse effect characterized by recurrent, sharply demarcated, red to livid maculae that evolve into plaque or bullae and erosions at the same skin or mucosal site after every intake of the causative drug. ${ }^{1,2}$ Common locations are the lips, oral mucosa, hands, geni- 